\title{
SIMULACIÓN MEDIANTE DINÁMICA MOLECULAR DE LOS CAMBIOS ESTRUCTURALES DURANTE LA COLISIÓN DE NANOPARTÍCULAS DE COBRE
}

\author{
Betty Copa ${ }^{\mathrm{a}^{*}}$, Justo Rojas T. ${ }^{\mathrm{a}, \mathrm{b}}$. \\ ${ }^{a}$ Facultad de Ciencias Físicas, Universidad Nacional Mayor de San Marcos Ap. Postal 14-0149, Lima 14, Perú. \\ ${ }^{b}$ División de Materiales, Instituto Peruano de Energía Nuclear, Av. Canadá No 1470, Lima 41,Perú.
}

(Recibido Agosto 04, 2009; Aceptado Setiembre 29,2009)

\begin{abstract}
Molecular dynamics simulations with embedded-atom potential (EAM) have been performed to study the energetic and structural changes during the collision and coalescence of two $\mathrm{Cu}_{\mathrm{n}}$ nanoparticles. We simulated collision of nanoparticles at several temperatures below the melting point and with different impact energy. Analyzing the potential energy change during the collision we identify three clearly defined stages. The pair correlation function and the pair analysis technique are used to reveal the structural changes in the collision process. The variation in the time of the population of different pairs has been quantified, being observed diverse structural transformations. During the collision of two equal icosahedral nanoparticles $\left(\mathrm{Cu}_{55}\right)$ has been observed different behavior of 1551 pairs depending on the impact velocity.
\end{abstract}

PACS: 02.70.Ns; 31.15.at; 31.15.xv

Keywords: Collision, Nanoparticles, Coalescence, Molecular dynamics.

\section{Resumen}

Mediante la simulación con el método de Dinámica Molecular usando el potencial de interacción de tipo EAM se ha estudiado los aspectos energéticos y estructurales durante la colisión de dos nanopartículas de $\mathrm{Cu}_{\mathrm{n}}$. Simulamos la colisión de las nanopartículas a diferentes temperaturas debajo del punto de fusión y con diferentes velocidades de impacto. Analizando la variación de la energía potencial durante la colisión identificamos tres etapas claramente definidas. La información sobre los cambios estructurales durante la colisión se obtiene mediante la función de correlación par y la técnica de análisis de pares. Se ha cuantificado la variación en el tiempo de la población de diferentes pares, observándose diversas transformaciones estructurales. Luego se nota una reorganización de átomos hacia una forma más estable mediante la liberación de energía. Durante la colisión de dos nanopartículas icosaedrales iguales $\left(\mathrm{Cu}_{55}\right)$ se ha observado diferente comportamiento de los pares vecinos 1551 dependiendo de la velocidad de impacto.

Palabras claves: Colisión, Nanopartículas, coalescencia, Dinámica molecular.

\section{Introducción}

En la actualidad las nanopartículas (NP) metálicas son objeto de intensa investigación tanto experimental como teórica, principalmente por el hecho que sus propiedades presentan fuerte dependencia del tamaño y también porque representan los elementos de construcción de nanoestructuras y nanomateriales [1-3]. La colisión de los clusters entre si, así como con el substrato u otra superficie, es un fenómeno interesante y juega un papel importante en varios procesos a nanoescala [4]. Por ejemplo durante la obtención de nanopartículas a partir de la fase gaseosa [5,6], el depósito sobre un substrato para obtener películas nanoestructuradas [7] y en los procesos de coalescencia y agregación de las nanopartículas [8,9]. Dependiendo de la razón entre los tiempos de colisión y coalescencia, los productos finales pueden ser partículas esféricas relativamente grandes o un aglomerado de pequeñas partículas con estructura semejante a la fractal [9]. Diferentes aspectos de los procesos de colisión y coalescencia de clusters tales como la dinámica, cinética, energéticos y térmicos fueron estudiados ampliamente [10-14]. Sin embargo, acerca de los detalles de los cambios

\footnotetext{
*Correspondig author.e-mail: bettycopa@gmail.com
} 
estructurales durante la colisión y coalescencia hay poca información. Además, el estudio experimental de los mencionados procesos a escala atómica se encuentra con serias dificultades [10].

El objetivo del presente trabajo es estudiar los cambios estructurales durante la colisión de diferentes clusters de cobre usando el método de Dinámica Molecular (DM).

\section{Modelo y Simulación}

\subsection{Método de dinámica molecular}

El método de DM, que consiste en la solución numérica del sistema de ecuaciones de movimiento de $\mathrm{N}$ átomos o moléculas que interactúan con cierto potencial, es particularmente apropiado para estudiar en detalle los procesos a nivel atómico. Los fundamentos del método de DM están descritos en muchos trabajos, ver por ejemplo [15]. En la simulación usamos el potencial del modelo del átomo insertado EAM (Embedded Atom Model) [16,17]. Este tipo de potencial es usado ampliamente para la simulación atomística de metales y aleaciones con DM $[8,9,18]$

En el modelo de potencial EAM la energía total del sistema se define como:

$U_{t o t}=\frac{1}{2} \sum_{i \neq j} V\left(r_{i j}\right)+\sum_{i} F\left(\overline{\rho_{i}}\right)$

donde $V\left(r_{i j}\right)$ es la energía de interacción de pares como función de la distancia $r_{i j}$ entre los átomos $i$ y $j$. El segundo termino es la energía de inserción que depende de la densidad electrónica media, definida por,

$$
\overline{\rho_{i}}=\sum_{j \neq i} \rho\left(r_{i j}\right)
$$

\subsection{Detalles de la simulación}

En el presente trabajo se han estudiado la colisión de los siguientes clusters: $\mathrm{Cu}_{55}+\mathrm{Cu}_{55} \rightarrow \mathrm{Cu}_{110}$ (sistema $\mathrm{S} 1$ ), $\mathrm{Cu}_{55}+\mathrm{Cu}_{736} \rightarrow \mathrm{Cu}_{791}(\mathrm{~S} 2)$.

Para el estudio de la colisión de dos nanopartículas, estas se ubican inicialmente a cierta distancia entre los centros de masa, en el eje $x$ del sistema de coordenadas; $2.0 \mathrm{~nm}$ para el sistema $\mathrm{S} 1 \mathrm{y}$ $2.5 \mathrm{~nm}$ en el caso del sistema S2. Estas distancias se han escogido de tal manera que la separación entre los átomos en los bordes cercanos de las NP sea mayor que la distancia de corte $r_{c}$, donde $r_{c}$ está definido tal que $V\left(r_{i j}\right)=0$ para distancias $r_{i j}$ mayores que $r_{c \text {. }}$
El tipo de simulación canónica o micro-canónica en sistemas de nanoescala juega un papel importante. En el presente trabajo realizamos la simulación canónico o isotérmico (NVT), es decir se mantienen constantes el número de átomos $(\mathrm{N})$, el volumen $(\mathrm{V})$ y la temperatura (T). Las dimensiones de la caja de simulación son $10 \times 5 \times 5 \mathrm{~nm}^{3}$ y no se aplican condiciones de frontera periódicas.

El programa de DM que usamos para la simulación es el XMD [19]. En este programa, la integración numérica del sistema de ecuaciones de movimiento se realiza mediante el algoritmo predictor-corrector de quinto orden. El paso de integración para el cobre fue de $1.6 \mathrm{fs}$. La función de energía EAM (1) viene en forma tabulada, mediante la cual se calculan las fuerzas resultantes que actúan sobre cada átomo en cada instante de tiempo $t_{i}$. Conociendo la fuerza se calcula la velocidad y las nuevas posiciones en el siguiente instante de tiempo.

Al comienzo de la simulación se permite la relajación del sistema formado por las dos NP a la temperatura requerida durante 5000 pasos, con los centros de masas de los NP inmóviles. Luego se comunica velocidades iniciales adicionales a cada NP en sentidos opuestos a lo largo del eje $x$. Las magnitudes de las velocidades de cada NP se calculan de tal manera que el momento lineal total del sistema sea igual a cero. Durante la simulación los datos acerca de la trayectoria de fase, es decir posiciones y velocidades, así como la energía total, potencial y cinética, son almacenados para el posterior procesamiento.

\subsection{Técnicas de análisis.}

Para el análisis de los cambios estructurales se calcula la función de correlación par (FCP), definida mediante [20],

$g(r)=\frac{<n_{i}(r, r+\Delta r)>}{4 \pi r^{2} \Delta r} \frac{V}{N}$

donde $\left\langle n_{i}(r+\Delta r>\right.$ es el número promedio de átomos en una capa esférica de radio $r$ y $r+\Delta r$ al rededor de un átomo tomado como centro.

Adicionalmente, para el análisis mas detallado de los cambios estructurales se usa la técnica de análisis de vecinos comunes de Honeycut et al [21]. En esta técnica, la estructura atómica de sólidos y líquidos se caracteriza mediante 4 índices $i, j, m, n$. El primer índice $i$ es igual a la unidad si el par de átomos son considerados como primeros vecinos y 2 en caso contrario. El segundo índice $j$ indica el número de vecinos comunes del par considerado, mientras que $m$ indica el número de enlaces entre los vecinos comunes. La Fig. 1 muestra algunas configuraciones locales o simetrías más comunes en las NP 
estudiadas y sus respectivos indexes. Los índices 1421 y 1422 caracterizan a la simetría $f c c$ y $h c p$ respectivamente. La estructura icosaedral se caracteriza con los índices 1551, mientras que la simetría $f c c$ y icosaedral distorsionadas se caracterizan mediante 1431 y 1541 , respectivamente.

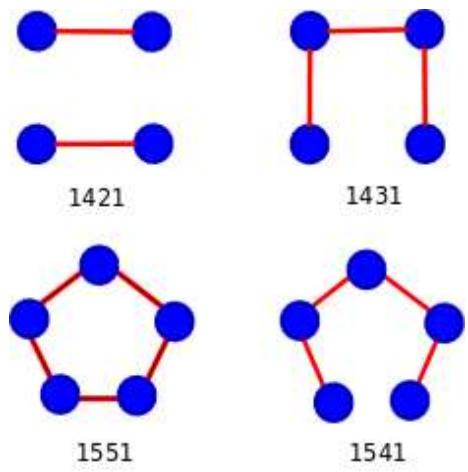

Fig. 1. Representación compacta de algunos pares. En este caso el par de átomos se encuentran en una línea perpendicular al plano y pasa por el centro de las figuras.

Para el procesamiento de los datos generados en la simulación se ha utilizado el programa CMD, desarrollado por Lobato et al [22], el cual, entre otras utilidades, permite calcular la FCP, la población relativa del número de diferentes pares con determinados vecindades.

\section{Resultados y Discusión}

Las NP de $\mathrm{Cu}_{55}$ y $\mathrm{Cu}_{147}$ optimizadas forman estructura icosaedral y pertenecen al grupo de los llamados números mágicos, que son particularmente estables. En cambio, en la nanopartícula $\mathrm{Cu}_{736}$ la estructura $f c c$ es la simetría con mayor población y tiene forma de octaedro truncado. En la tabla 1 se presentan los valores de población relativa de los principales pares ligados en las NP optimizadas.

Tabla 1. Índices principales en las NP en el estado inicial.

\begin{tabular}{llll}
\hline $\mathrm{i}, \mathrm{j}, \mathrm{k}, \mathrm{m}$ & $\mathrm{N}=55$ & $\mathrm{~N}=147$ & $\mathrm{~N}=736$ \\
\hline 1321 & 0.25 & 0.129 & 0.024 \\
1421 & 0 & 0.017 & 0.68 \\
1422 & 0.38 & 0.387 & 0.062 \\
1431 & 0 & 0 & 0.015 \\
1541 & 0 & 0 & 0.011 \\
1551 & 0.10 & 0.051 & 0 \\
\hline
\end{tabular}

La energía potencial y total del sistema sufre cambios importantes durante el proceso de colisión de las NP. En la Fig. 2 se presenta, como ejemplo, la variación de la energía potencial durante la colisión de dos NP idénticas de $\mathrm{Cu}_{55}$ a temperatura de $300 \mathrm{~K}$. La Fig.2a corresponde a una velocidad de colisión entre las NP de $1.6 \mathrm{~nm} / \mathrm{ps}$, lo que es equivalente a una energía cinética de traslación de $0.42 \mathrm{eV}$ por átomo.

La simulación de la colisión se ha realizado a diferentes temperaturas y velocidades iniciales. En todos los casos la variación de la energía potencial muestra cualitativamente 3 etapas bien marcadas. En la primera etapa, cuando las NP ingresan al campo de acción de una con respecto a la otra, su energía potencial disminuye rápidamente durante un intervalo de tiempo aproximadamente de 0.5 ps. Luego de la colisión directa, cuando las fuerzas de repulsión entre las partes en contacto empiezan prevalecer y empieza la perdida del orden atómico inicial, la energía potencial crece hasta alcanzar un valor máximo que corresponde, aparentemente, a la máxima deformación o desorden en la distribución atómica. El hecho de que a mayor energía cinética de impacto (Fig.2b) es mayor el crecimiento de la energía potencial en esta etapa, respalda la suposición de que el incremento de la energía en esta etapa está relacionado con la deformación de la red. En la tercera etapa la energía empieza nuevamente a disminuir debido al reordena miento atómico.
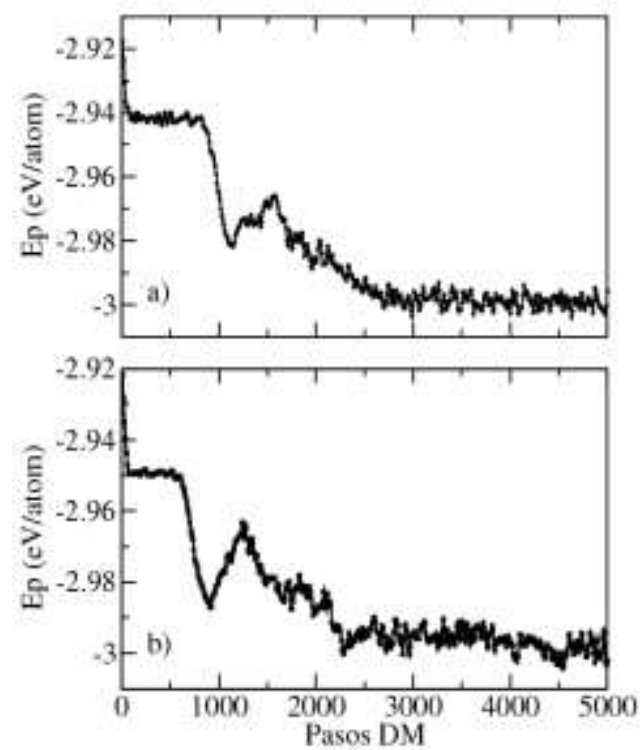

Fig. 2. Variación de la energía potencial como función del número de pasos de DM para el sistema $\mathrm{S} 1$ a $300 \mathrm{~K}$; a) $\varepsilon=0.42 \mathrm{eV} / \mathrm{atom}, \mathrm{b}) \varepsilon=3.78 \mathrm{eV} / \mathrm{atom}$

La Fig.3 ilustra cualitativamente los cambios en la distribución atómica correspondientes a diferentes instantes de tiempo. Los átomos de $\mathrm{Cu}$ pertenecientes a cada NP se muestran con diferentes colores solamente con fines de ilustración. Prestando atención a la morfología externa notamos que luego 
de la colisión el sistema compuesto coalece y tiende a adquirir la forma esférica, es decir la reducción de la superficie se manifiesta como la fuerza motriz en las etapas posteriores a la colisión, la cual es descrito por la ley de Koch y Friedlander [23]. En gran parte la disminución de la energía potencial en la tercera etapa se debe precisamente a la reducción de la energía superficial.
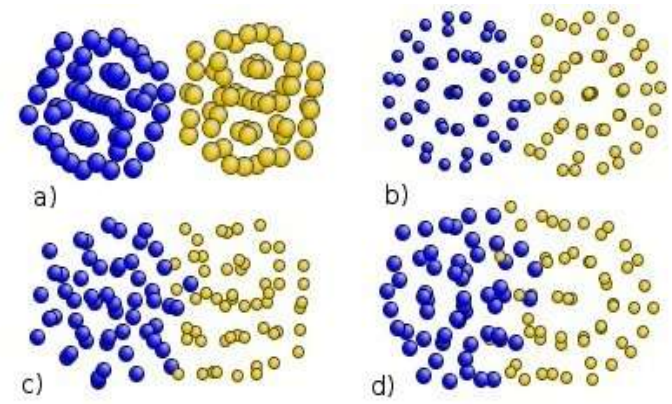

Fig. 3. Configuraciones atómicas durante la colisión de dos $\mathrm{NP}$ de $\mathrm{Cu}_{55}$ a temperatura $300 \mathrm{~K}$ y $\varepsilon=3.78 \mathrm{eV}$ por átomo, correspondientes a diferentes instantes de tiempo (pasos DM): a) 800 , b) 1000 , c) 1200 y d) 5000 .

En la Fig.4 se presenta el monitoreo del proceso de colisión de $\mathrm{NP}$ de $\mathrm{Cu}_{55}+\mathrm{Cu}_{736}$, que se realizó a temperatura de $300 \mathrm{~K}$. En este caso la NP $\mathrm{Cu}_{55}$ tiene una velocidad inicial igual a $1.6 \mathrm{~nm} / \mathrm{ps}$ mientras que la NP $\mathrm{Cu}_{736}-0.119 \mathrm{~nm} / \mathrm{ps}$.

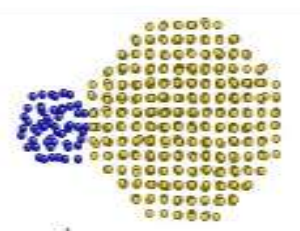

a)

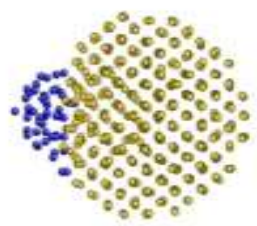

c)

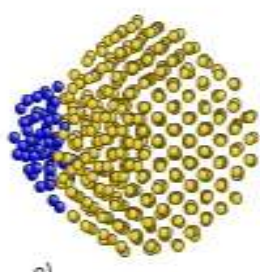

e)

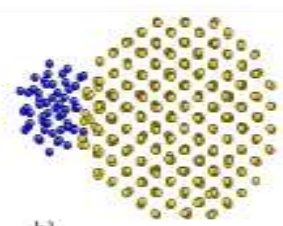

b)

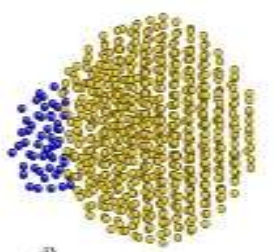

d)

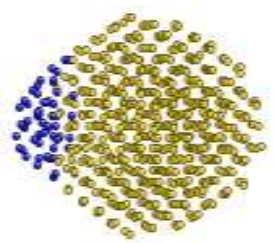

f)
Fig. 4. Evolución temporal de la colisión de NP de $\mathrm{Cu}_{55}+$ $\mathrm{Cu}_{736}$ a temperatura $600 \mathrm{~K}$ y parámetro de energía $\varepsilon=0.84$ $\mathrm{eV}$ por átomo, correspondientes a los instantes de tiempo (pasos DM) de a) 400, b) 500, c) 800 , d) 1000 , e) 1500, f) 5000.
En todos los casos estudiados luego de la colisión se forma una única NP, es decir que la colisión es puramente inelástica y no se observa restitución, a diferencia de lo que se observa en el caso de colisión con velocidades del orden de $0.004 \mathrm{~nm} / \mathrm{ps}$ [13]. En el caso del sistema S2 se ha observado que al final del proceso de colisión y coalescencia se forma un único "nanocristal".

La Fig.5 ilustra las funciones de correlación par de los sistemas S1 y S2 a temperatura de $300 \mathrm{~K}$.

Es conocido que la presencia de picos agudos en la función representa una estructura mas ordenada, mientras que el ensanchamiento del pico corresponde a una estructura mas desordenada. Se aprecia que en el caso del sistema S1 (Fig.5a) al final de la segunda etapa, mencionado mas arriba, la distribución espacial de los átomos es casi amorfa, mientras que en el caso del sistema S2 (Fig.5b) la FCP no muestra la amorfización, a pesar de que en realidad en la región de interfase si ocurre el desordenamiento, como se puede apreciar mediante la visualización de las configuraciones respectivas (ver, por ejemplo la Fig.4c) Esta aparente contradicción se debe a que la FCP se calcula mediante la pro-mediación por todo el sistema.

En los sistemas donde la diferencia de tamaños de las NP es significativa, el desordenamiento en una región relativamente pequeña no se manifiesta del todo cuando se hace el promedio por todo el sistema.
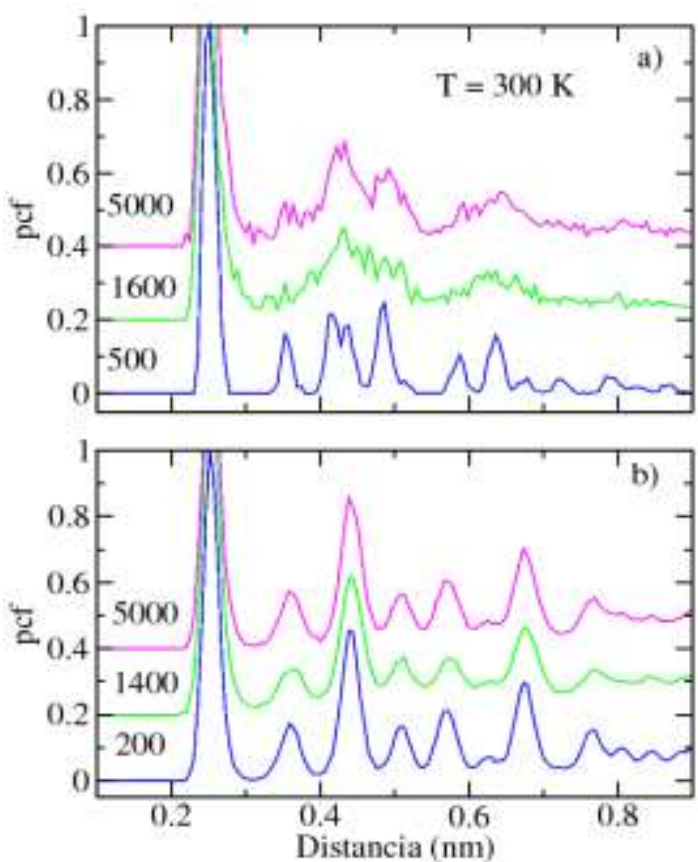

Fig. 5. Funciones de correlación par a temperatura $300 \mathrm{~K}$ correspondientes a diferentes instantes de tiempo: a) sistema $\mathrm{Cu}_{55}+\mathrm{Cu}_{55}$ con $\varepsilon=0.42 \mathrm{eV} /$ atom, b) sistema $\mathrm{Cu}_{55}+$ $\mathrm{Cu}_{736}$ y $\varepsilon=0.21 \mathrm{eV} /$ atom. 
La técnica de análisis de pares y su entorno nos permite obtener información mas detallada acerca de los posibles cambios estructurales que ocurren durante los procesos de colisión. A partir de los datos obtenidos durante la simulación se han analizado varios índices que caracterizan las diferentes simetrías.

De la Fig. 6a se nota que durante los primeros 1000 pasos no ocurren cambios notorios, luego la abundancia de los pares 1422, que caracterizan a la simetría $h c p$, disminuye sustancialmente, mientras que simultáneamente los pares 1421 y 1431 aumentan, sumando entre ambos un incremento aproximadamente de $20 \%$. Estos resultados indican como un probable mecanismo que las estructuras locales $h c p$ se transforman en parte a $f c c$ con distorsiones y $f c c$ perfectos.

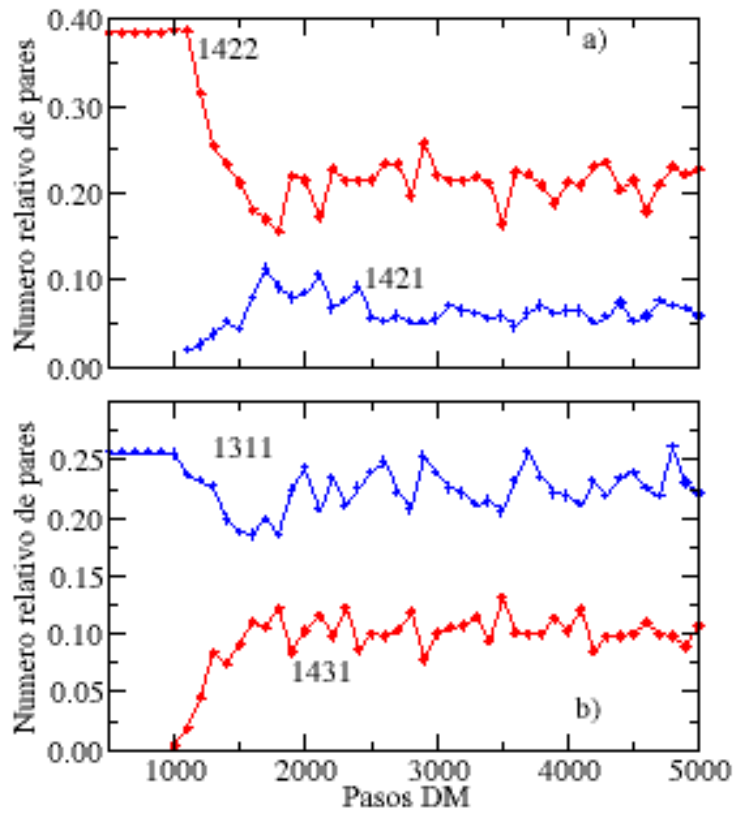

Fig. 6. Variación con el tiempo de la población de algunos pares ligados durante la colisión de los nanoclusters $\mathrm{Cu}_{55}+\mathrm{Cu}_{55}$ a temperatura $300 \mathrm{~K}$ y $\varepsilon=0.42 \mathrm{eV} /$ atomo

La Fig.7 muestra un comportamiento interesante de la población de los pares 1551, obtenidos durante la colisión de nanoclusters idénticos pero con dos parámetros de energía que se diferencian significativamente. Durante la colisión con velocidad relativamente mas baja $\mathrm{v} 1=1.6 \mathrm{~nm} / \mathrm{ps} \quad(\varepsilon=0.42$ eV/atom) la población de los pares 1551 disminuye en las dos primeras etapas, mientras que en el caso de colisión con mayor velocidad $\mathrm{v} 2=4.8 \mathrm{~nm} / \mathrm{ps}$, entre la segunda y comienzos de la tercera etapa, presenta un pico bien definido. La reproducibilidad de este pico en diferentes corridas es bastante alta. Se sabe que los pares 1551 se encuentran en abundancia en líquidos superenfriados y amorfos. Por tanto la formación del pico sería un indicio de amorfización durante un tiempo bastante corto.

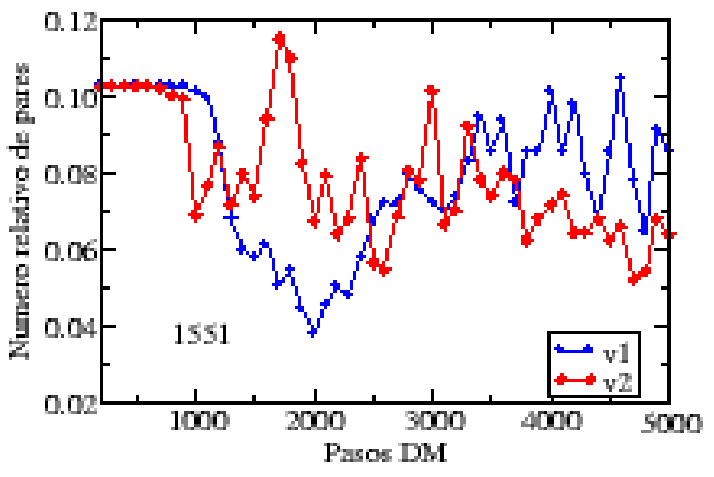

Fig. 7. Variación con el tiempo de la población relativa de los pares 1551 durante la colisión de los nanoclusters $\mathrm{Cu}_{55}+$ $\mathrm{Cu}_{55}$ a temperatura de 300. v1 corresponde a energía $\varepsilon=0.42 \mathrm{eV} /$ atom, mientras que v2 a $\varepsilon=3.78 \mathrm{eV} /$ atom.

La Fig.8 muestra, para el caso del sistema S2, la influencia de la velocidad de colisión en el grado de variación de la estructura. A mayor energía de colisión es mayor la variación de la población de los pares, en particular de los pares con índices 1421 y 1541. En este caso la estructura $f c c$ se transforma en parte a icosaedral puro e icosaedral con distorsiones.
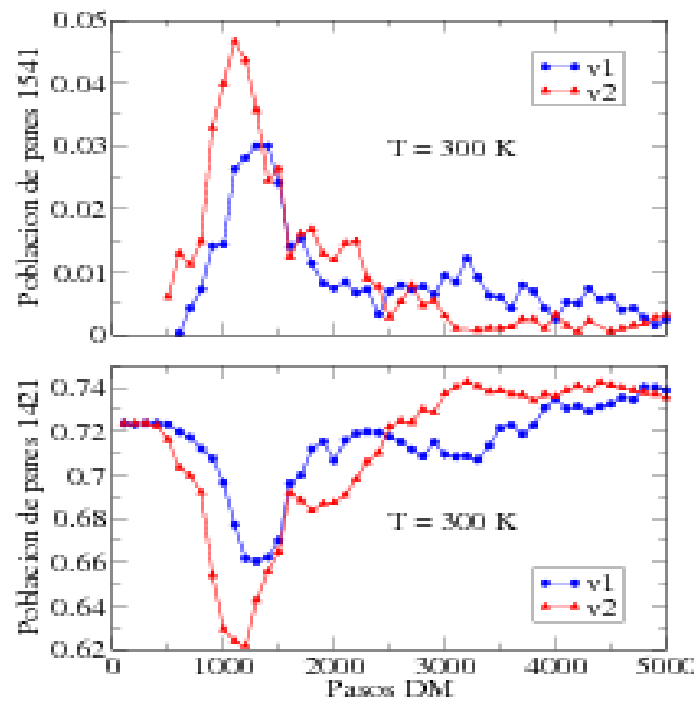

Fig. 8. Variación de la población de los pares 1541 y 1521 en la colisión de $\mathrm{Cu}_{55}+\mathrm{Cu}_{736}$ con v1=1.6 nm/ps y v2=3.2 $\mathrm{nm} / \mathrm{ps}$. 


\section{Conclusiones}

Se ha estudiado mediante la simulación con el método de DM clásica las particularidades de variación de la energía y estructura atómica durante los procesos de colisión y coalescencia de dos NP de $\mathrm{Cu}$. Se ha establecido tres etapas bien definidas en la variación de la energía potencial durante la simulación en condiciones isotérmicas. Dependiendo de las condiciones de colisión, las magnitudes de los mínimos y máximos en la energía potencial así como los intervalos de duración de cada etapa son diferentes. El análisis de la función de correlación par muestra los diferentes grados de amorfización que ocurre a diferentes condiciones de temperatura y energías de colisión. Se ha observado, en particular, que durante la colisión de NP icosaedrales de igual tamaño la población de los pares 1551 muestra un incremento notorio en la segunda etapa cuando la energía de colisión es alta, lo que no se observa a energías de colisión menores.

\section{Agradecimientos}

Los autores agradecen a Iván Lobato por permitir utilizar el programa CMD y al Concejo Superior de Investigación de la UNMSM por el soporte financiero parcial mediante el proyecto $\mathrm{N}^{\circ}$ 051301061.

\section{References}

[1]. P. Jansen, Phys. Rev B, 23 (2004) 345-356.

[2]. W. Eberhardt, Surface Science 500 (2002) 242 270.

[3]. P. Mendes, Yu Chen, R. E Palmer, K. Nikitin, D. Fitzmaurice and J. A Preece, J. Phys.: Condens. Matter 15 (2003) S3047-S3063.

[4]. M. R. Zachariah and Michael Carrier. J. Aerosol Sci. 30 (9) (1999) 1139-1151.
[5]. T. K. Sindhu, R. Sarathi and S.R. Chacravarthy, Nanotechnology, 19 (2008) 025703.

[6]. L. Zhang, M.B. Ranade and J.W. Gentr, Aerosol Science 33 (2002) 1559-1575.

[7]. P. Jensen, N. Combe, Computational Materials Science 24 (2002) 78-87.

[8]. L. J. Lewis, P. Jensen, and J. Barret, Phys. Rev. $B, 56$ (1997) 2248.

[9]. K. E. Lehtinen, and M. R. Zachariah, Phys. Rev. B 63 (2001) 205402.

[10]. M. Kalweit, D. Drikakis, Phys. Rev. B 74 (2006) 235415.

[11]. T. Hawa and M.R. Zachariah, Aerosol Science 37 (2006) 1-15.

[12]. K. E. Lehtinen and M. R. Zachariah, Aerosol Science 33 (2002) 357-368.

[13]. S. Sato, Da-Ren Chen David and Y. H. Pui, Aerosol and air quality 7 (3) (2007) 278-303.

[14]. J. Rojas and Ch. Rojas, Internet Electron J. Nanocs. Moletrón 4 (2006) 805-814.

[15]. K. Binder, J. Horbach, W. Kob, W. Paul and F. Varnik, J. Phys.: Condens. Matter 16 (2004) S429-S453.

[16]. M.S Daw and M.I Baskes, Phys. Rev. B, 29(1984) 6443.

[17]. R. A. Johnson, Phys. Rev. B, 39(1989) 1255412559.

[18]. S. J. Zhao, S.Q. Wang, Z.Q. Yang and H.Q. Ye, J. Phys. Condens. Matter, 13 (2001) 80618069.

[19]. J. Rifkin: XMD-2.5.32 Molecular Dynamics Program (Univ. of Connecticut, 2003)

[20]. F.H. Stillinger and T.A Weber, Phys. Rev. A, 25 (1982) 978

[21]. J. D. Honeycut and H. C. Anderson, J. Phys. Chem.91 (1987) 4950.

[22]. I. Lobato, J. Rojas, Rev. Inv. Fis. 11 (1) (2008) 43-48.

[23]. W. Koch and S.K. Friedlander, J. Colloid Interface Sci. 140 (1990) 419- 427. 